\title{
Computed tomography accelerates staging in patients with Merkel cell carcinoma
}

\author{
Elisabeth Foki ${ }^{1} \cdot$ Alexandra Fochtmann-Frana ${ }^{2} \cdot$ Georg Haymerle ${ }^{1} \cdot$ Stefan Nemec $^{3} \cdot$ Benjamin Loader $^{4}$. \\ Christos Perisanidis ${ }^{5}$. Boban M. Erovic ${ }^{1}$
}

Received: 3 August 2018 / Accepted: 15 September 2018 / Published online: 28 September 2018

(C) The Author(s) 2018

\begin{abstract}
Purpose No imaging algorithms for diagnostic imaging in patients suffering from Merkel cell carcinoma (MCC) have been established so far and thus staging work-up is challenging. Long presentation-to-treatment intervals determine further treatment course and, consequently, have an impact on clinical outcome in patients with MCC.

Methods In this retrospective study, diagnostic imaging of $37 \mathrm{MCC}$ patients was analyzed. CT, ultrasound, and PET/PETCT imaging for primary staging work-up with time frames from patients' initial presentation and imaging until completion of tumor staging were analyzed.

Results Tumor staging could be completed earlier when (1) less examinations (35 vs. 42 days) were carried out or (2) computed tomography was used as the initial imaging modality (28 vs. 35 days). Furthermore, CT imaging, when used as the initial imaging study, was linked to less follow-up imaging (3 vs. 6).

Conclusion Computed tomography as the first-staging imaging technique in MCC patients leads to less follow-up studies and fastest completion of tumor staging.
\end{abstract}

Keywords Imaging $\cdot$ Diagnosis $\cdot$ Cancer $\cdot$ Skin $\cdot$ Ultrasound $\cdot$ Computerized tomography

\section{Introduction}

Merkel cell carcinoma (MCC) is a rare and highly aggressive neuroendocrine tumor of the skin with a significantly higher mortality rate than melanomas [1]. The previous results showed an age-adapted threefold increase of MCC incidence

Boban M. Erovic

boban.erovic@meduniwien.ac.at

1 Department of Otorhinolaryngology, Head and Neck Surgery, Medical University of Vienna, Währinger Gürtel 18-20, 1090 Vienna, Austria

2 Department of Surgery, Clinical Division of Plastic and Reconstructive Surgery, Medical University of Vienna, Vienna, Austria

3 Department of Radiology, Division of Neuroradiology and Musculoskeletal Radiology, Medical University of Vienna, Vienna, Austria

4 Department of Otorhinolaryngology, Head and Neck Surgery, Rudolfstiftung Teaching Hospital, Vienna, Austria

5 Department of Oral and Maxillofacial Surgery, Medical University of Vienna, Vienna, Austria between 1986 and 2001 [2]. Thus, diagnostic work-up, histopathologic verification, and disease management of patients with Merkel cell carcinoma is becoming more important.

Due to its similarity to benign skin tumors, the diagnosis of Merkel cell carcinoma can be challenging [3]. Patients typically present with new, rapidly developing skin lesions. MCC appears characteristically as firm, non-tender, domeshaped, red, purple, or violet nodule $[4,5]$. The overlying skin is smooth and shiny, sometimes exhibiting ulcerative, acneiform, or telangiectatic signs [4, 6]. It affects mainly the sun-exposed area of the head and neck [7,8], each at a rate of $40 \%$ [9]. Clinically MCC spreads rapidly to regional lymph nodes and in 50\% distant metastasis occur, predominantly in the liver, bone, brain, and the lungs $[6,7]$. The 5 -year survival rate for patients suffering from a primary tumor without metastasis is $75 \%$, whereas it is $59 \%$ for patients suffering from local recurrence and/or lymph-node metastasis [9]. The 2-year disease-specific survival rate is dismal, with an $11 \%$ estimation, when distant metastatic disease is detected [9]. Due to the aggressive behavior of this tumor, rapid diagnosis and staging work-up are essential for 
treatment, since prolonged waiting times are linked to worse clinical outcome in patients with cancer [10-13].

However, still, to date, there is sparse literature on imaging algorithms or widely accepted guidelines for imaging in patients with Merkel cell carcinoma [3, 4]. Recently published literature looked in particular at the diagnostic impact of various imaging methods such as computed tomography, sonography of the lymph nodes, and PET/PET-CT [3, 4, 7].

Hawryluk and colleagues recommended 18-FDG PET for the detection of bone metastasis [14]. However, it has limited value for detection of liver metastasis. In the detection of lymph-node involvement, PET-CT has a sensitivity and specificity of $83 \%$ and $95 \%$, respectively [14]. In a study of Concannon, it altered staging in 33\% of all cases as it was more sensitive than clinical examination, CT, as well as MRI [15].

Peloschek and colleagues recommended ultrasonography as the initial imaging method in staging of patients with Merkel cell carcinoma. In easy accessible lymph-node regions, ultrasonography is cost-effective and highly accurate [7]. In the same study, the authors could show that computed tomography has a specificity of $96.2 \%$ and a sensitivity of $89.1 \%$ for imaging of lymph-node involvement and distant metastasis in MCC patients [7]. A meta-analysis of Liao showed that $\mathrm{CT}$ and ultrasonography have a similar sensitivity, but CT is superior in regards to specificity in lymph-node mapping of the head and neck area compared to the ultrasound [16].

Summarizing the literature of imaging of MCC, the major diagnostic tools for staging are CT, lymph-node sonography, and PET or PET-CT. The objective of this study was, hence, to analyze whether one of the aforementioned diagnostic methods or the overall number of imaging techniques performed influences time and accuracy of MCC staging.

\section{Materials and methods}

\section{Patients}

Retrospective data of 37 patients were available ( 20 females and 17 males). Mean and median age at the time of first diagnosis of Merkel cell carcinoma was 72.5 and 76 years, respectively (range 46-87 years). All patients were treated at the Departments of Dermatology and Otorhinolaryngology, Head and Neck Surgery, at the Medical University of Vienna. Only patients diagnosed with a new and untreated Merkel cell carcinoma between 1994 and 2012 were included in this study. Demographic, clinical, and pathological data were obtained from hospital records. Approval of the institutional research ethics board was retrieved before performing this study (REB\# 1798/2013).

\section{Statistics}

Demographic and pathologic data were summarized using descriptive statistics. Outcome measures were number of radiologic examinations and time interval for imaging and completion of tumor staging. In particular, the time frame between primary presentation and first imaging (FI) and the total time interval between primary presentation and completion of radiologic staging before treatment onset (CS) were determined (days). Imaging studies were counted as baseline examinations if the examination was performed promptly to the first physicians' visit either in an outpatient setting or in a hospital and if it was realized before the onset of treatment. An imaging study was defined as imaging of a predefined part of the body, e.g., the cervical lymph nodes or computed tomography of the abdomen. As a consequence, we counted three examinations when (1) computed tomography of the head and neck, (2) thorax, and (3) abdomen was done in one patient until staging was completed.

During the staging process, the majority of our patients were seen more than once, and subsequently, imaging studies were performed until the final staging was completed. Histologic data were not included in our data evaluation.

Demographic and pathologic data were summarized using descriptive statistics. Mean, median, and standard deviation were calculated. False-negative and false-positive results of the conducted examinations were evaluated in concordance with the available reports. Examinations were stratified according to their accuracy and it was taken into concern if staging was altered during the course of treatment. However, if staging was altered after 6 months after the first diagnosis, it was counted as recurrent disease. Sensitivity and specificity of the imaging methods were calculated. Cost-effectiveness of conducted examinations was calculated according to the routine costs in our environment using one-way analysis of variance and $t$ test, respectively.

\section{Staging}

Seventeen, fourteen, and two patients with stage I (46\%), stage II $(37.8 \%)$, and stage III $(5.4 \%)$ diseases were included in this study, respectively. According to the seventh edition of the AJCC cancer system, stage I is defined as tumor size $\leq 2 \mathrm{~cm}$ across, stage II is defined as tumor size $>2 \mathrm{~cm}$ across, and stage III is defined as any size of primary tumor invading nearby lymph nodes. In 4 (10.8\%) of 37 patients, no primary tumor was found. Those patients were defined as patients with cancer of unknown primary. In $51.4 \%(n=19)$, the primary tumor site was within the 
head and neck areas, in 27\% $(n=10)$ on the upper and lower limbs, and in $21.6 \%(n=8)$ the trunk. Four $(10.8 \%)$ patients suffered from recurrent disease.

Surgery was performed in $46 \%(n=17)$, primary radiation treatment in $5.4 \%(n=2)$, surgery and subsequent radiation treatment in $40.5 \%(n=15)$, adjuvant chemoradiation therapy in $5.4 \%(n=2)$, and surgery followed by chemotherapy in $2.7 \%(n=1)$ patients. Twenty-eight patients were treated with curative intention $(75.7 \%)$ and nine patients were treated with palliative intention $(24.3 \%)$. Twenty-four patients $(64.9 \%)$ had to undergo re-resection to achieve negative resection margins.

\section{Imaging}

Thirty-seven patients underwent a total of 168 radiologic examinations before staging was confirmed. The mean number of investigations per patient was 4.5 (range 2-8). In $78.4 \%$ of all patients, one or more examinations with computed tomography of the neurocranium, thorax, or abdomen were performed $(n=29)$. Contrast agent-containing iodine was used routinely. Sonography of the cervical, axillary, and inguinal lymph nodes was performed in $62.2 \%$ of the patients $(n=23)$, and seven patients underwent a PET or PET-CT scan (19\%). Further imaging methods such as octreotide scintigraphy, MRI, or X-ray of the bones and lungs were sub-summarized as "various examinations" as they were performed in less than three patients.

\section{Results}

\section{Waiting times for all MCC patients}

The median time until the first imaging method was performed, and staging was completed was 19 and 35 days, respectively. The mean value in days for FI and CS was 25 (SD: 23 days), and 38 (SD: 21 days), respectively.

\section{Group stratification according to the count of examinations}

Patients who underwent four examinations or less $(n=19$, $51.4 \%$ ) or less until tumor staging was completed were stratified into group A and patients with five examinations or more $(n=18,48.6 \%)$ were stratified into group B (Fig. 1; Tables 1,2).

\section{Group A}

In group A ( $n=19,51.4 \%), 5$ patients underwent sonography of the lymph nodes (26.3\%) and 12 patients underwent
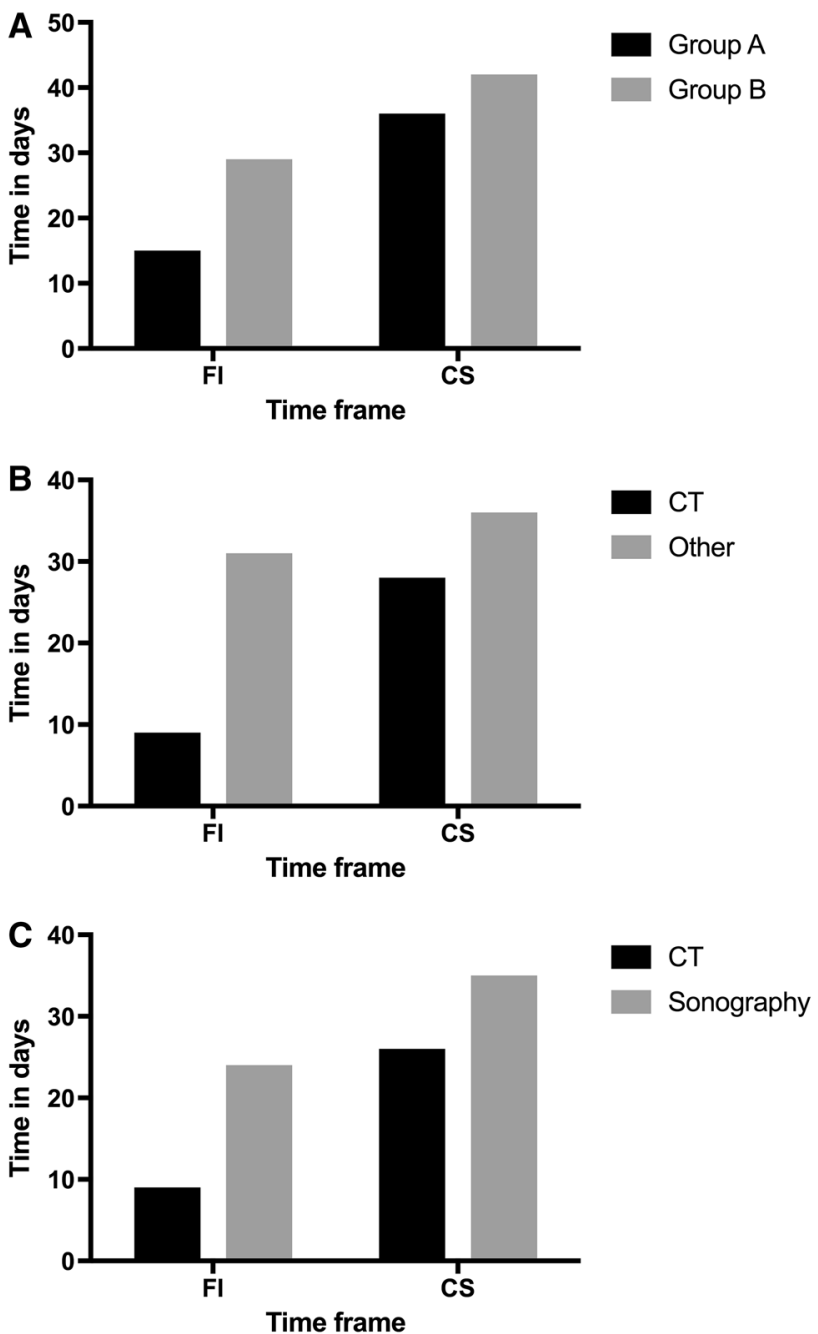

Fig. 1 Comparative data of patients according to number of imaging methods and imaging methods. a Patients undergoing a lower number of imaging methods (Group A) showed a shorter FI (15 vs. 29 days) and CS ( 35 vs. 42 days), respectively, compared to group B. b Performing a CT scan at the initial presentation of the patient led to a shorter FI ( 9 vs. 31 days) and CS ( 28 vs. 36 days) compared to other patients. c However, lymph-node sonography at the initial presentation but not computed tomography led to a longer FI ( 24 vs. 9 days) and CS ( 28 vs. 35 days). FI time interval from first consultation to first imaging, $C S$ time interval to completion of tumor staging

computed tomography for the initial staging work-up (63.2\%). Only two patients had lymph-node sonography combined with computed tomography (10.5\%). FI accounted for 15 days (median value; mean $=19$, SD: 16 days), whereas CS was completed after 35 days (median value; mean $=31$, SD: 17 days).

\section{Group B}

Group B consisted of 18 patients; 3 (11.1\%) patients underwent sonography of the lymph nodes and 2 had computed 
Table 1 Demographic data of 37 patients with Merkel cell carcinoma

\begin{tabular}{|c|c|c|c|c|c|c|c|}
\hline Patient & No. $^{a}$ & Group & $\mathrm{FI}^{\mathrm{b}}$ & $\mathrm{CS}^{\mathrm{c}}$ & Stage & Rec. $^{d}$ & $\mathrm{e}$ \\
\hline 1 & 3 & A & 20 & 36 & 1 & 0 & ANED \\
\hline 2 & 4 & A & 48 & 54 & 1 & 0 & DNED \\
\hline 3 & 7 & B & 36 & 54 & 2 & 0 & DOD \\
\hline 4 & 8 & B & 89 & 89 & 1 & 0 & ANED \\
\hline 5 & 7 & B & 19 & 28 & 1 & 0 & DNED \\
\hline 6 & 3 & A & 33 & 47 & 1 & 0 & DNED \\
\hline 7 & 7 & B & 40 & 40 & 1 & 0 & DNED \\
\hline 8 & 2 & A & 15 & 16 & 1 & 0 & DOD \\
\hline 9 & 5 & B & 82 & 82 & 1 & 1 & DOD \\
\hline 10 & 5 & B & 0 & 35 & 2 & 0 & DOD \\
\hline 11 & 5 & B & 10 & 51 & 2 & 0 & DNED \\
\hline 12 & 2 & A & 19 & 19 & 4 & 0 & ANED \\
\hline 13 & 4 & A & 49 & 49 & 1 & 0 & DNED \\
\hline 14 & 4 & A & 65 & 70 & 2 & 0 & ANED \\
\hline 15 & 4 & A & 35 & 35 & 1 & 0 & DNED \\
\hline 16 & 5 & B & 47 & 75 & 1 & 0 & DNED \\
\hline 17 & 5 & B & 3 & 10 & 4 & 0 & DOD \\
\hline 18 & 2 & A & 22 & 39 & 2 & 1 & DOD \\
\hline 19 & 3 & A & 2 & 33 & 1 & 0 & ANED \\
\hline 20 & 4 & A & 6 & 57 & 2 & 0 & DOD \\
\hline 21 & 3 & A & 41 & 41 & 3 & 0 & DOD \\
\hline 22 & 5 & B & 5 & 12 & 2 & 0 & DOD \\
\hline 23 & 6 & B & 27 & 21 & 2 & 0 & DOD \\
\hline 24 & 4 & A & 3 & 18 & 4 & 0 & DNED \\
\hline 25 & 2 & A & 10 & 50 & 2 & 0 & DOD \\
\hline 26 & 6 & B & 33 & 48 & 1 & 0 & DNED \\
\hline 27 & 2 & A & 35 & 35 & 2 & 0 & DNED \\
\hline 28 & 8 & B & 16 & 48 & 3 & 0 & DOD \\
\hline 29 & 6 & B & 34 & 35 & 1 & 0 & DNED \\
\hline 30 & 7 & B & 31 & 43 & 1 & 0 & DNED \\
\hline 31 & 7 & B & 11 & 29 & 2 & 1 & DOD \\
\hline 32 & 2 & A & 6 & 20 & 1 & 0 & DNED \\
\hline 33 & 2 & A & 122 & 122 & 1 & 0 & ANED \\
\hline 34 & 2 & A & 0 & 12 & 2 & 0 & DNED \\
\hline 35 & 6 & B & 78 & 78 & 2 & 0 & DNED \\
\hline 36 & 3 & A & 11 & 11 & 4 & 1 & DOD \\
\hline 37 & 8 & B & 7 & 29 & 2 & 0 & ANED \\
\hline
\end{tabular}

No. number of examinations, FI time interval from first consultation to first imaging, $C S$ time interval to completion of tumor staging

${ }^{\mathrm{a}}$ Number of examinations

${ }^{\mathrm{b}}$ Time from first consultation to first imaging

${ }^{\mathrm{c}}$ Time from first consultation to completion of tumor staging

${ }^{\mathrm{d}}$ Recurrent disease

${ }^{\text {e}}$ Status of survival: $A N E D$ alive with no evidence of disease, $D N E D$ died with no evidence of disease, $D O D$ died of disease tomography imaging (16.7\%) alone. In 13 (72.2\%) patients, ultrasound and CT scanning of the lymph-node basins was performed. In this group, patients completed FI and CS after
29 (median value; mean $=32$, SD: 27 days) and 42 (median value; mean $=45$, SD: 24 days), respectively. 
Table 2 Demographic data of patients with Merkel cell carcinoma according to imaging method

\begin{tabular}{|c|c|c|c|c|c|c|c|c|}
\hline Pat. & No. ${ }^{a}$ & Group & $\mathrm{FI}^{\mathrm{b}}$ & $\mathrm{CS}^{\mathrm{c}}$ & Sono $^{d}$ & CT & Combi $^{\mathrm{e}}$ & PET/CT \\
\hline 1 & 3 & A & 20 & 36 & 1 & 1 & 1 & 0 \\
\hline 2 & 4 & A & 48 & 54 & 1 & 0 & 0 & 0 \\
\hline 3 & 7 & B & 36 & 54 & 1 & 1 & 1 & 0 \\
\hline 4 & 8 & B & 89 & 89 & 1 & 1 & 1 & 0 \\
\hline 5 & 7 & B & 19 & 28 & 1 & 0 & 0 & 0 \\
\hline 6 & 3 & A & 33 & 47 & 0 & 1 & 0 & 1 \\
\hline 7 & 7 & B & 40 & 40 & 1 & 1 & 1 & 0 \\
\hline 8 & 2 & A & 15 & 16 & 0 & 1 & 0 & 0 \\
\hline 9 & 5 & B & 82 & 82 & 1 & 1 & 1 & 0 \\
\hline 10 & 5 & B & 0 & 35 & 0 & 1 & 0 & 0 \\
\hline 11 & 5 & B & 10 & 51 & 1 & 1 & 1 & 0 \\
\hline 12 & 2 & A & 19 & 19 & 0 & 1 & 0 & 0 \\
\hline 13 & 4 & A & 49 & 49 & 0 & 1 & 0 & 0 \\
\hline 14 & 4 & A & 65 & 70 & 0 & 1 & 0 & 0 \\
\hline 15 & 4 & A & 35 & 35 & 1 & 0 & 0 & 0 \\
\hline 16 & 5 & B & 47 & 75 & 1 & 1 & 1 & 0 \\
\hline 17 & 5 & B & 3 & 10 & 0 & 1 & 0 & 1 \\
\hline 18 & 2 & A & 22 & 39 & 0 & 1 & 0 & 0 \\
\hline 19 & 3 & A & 2 & 33 & 1 & 0 & 0 & 0 \\
\hline 20 & 4 & A & 6 & 57 & 0 & 1 & 0 & 0 \\
\hline 21 & 3 & A & 41 & 41 & 0 & 1 & 0 & 0 \\
\hline 22 & 5 & B & 5 & 12 & 1 & 1 & 1 & 0 \\
\hline 23 & 6 & B & 27 & 21 & 1 & 1 & 1 & 0 \\
\hline 24 & 4 & A & 3 & 18 & 0 & 1 & 0 & 1 \\
\hline 25 & 2 & A & 10 & 50 & 0 & 1 & 0 & 0 \\
\hline 26 & 6 & B & 33 & 48 & 1 & 0 & 0 & 0 \\
\hline 27 & 2 & A & 35 & 35 & 1 & 0 & 0 & 1 \\
\hline 28 & 8 & B & 16 & 48 & 1 & 0 & 0 & 1 \\
\hline 29 & 6 & B & 34 & 35 & 1 & 1 & 1 & 0 \\
\hline 30 & 7 & B & 31 & 43 & 1 & 1 & 1 & 0 \\
\hline 31 & 7 & B & 11 & 29 & 1 & 1 & 1 & 1 \\
\hline 32 & 2 & A & 6 & 20 & 0 & 1 & 0 & 0 \\
\hline 33 & 2 & A & 122 & 122 & 0 & 1 & 0 & 0 \\
\hline 34 & 2 & A & 0 & 12 & 1 & 0 & 0 & 0 \\
\hline 35 & 6 & B & 78 & 78 & 1 & 1 & 1 & 0 \\
\hline 36 & 3 & A & 11 & 11 & 1 & 1 & 1 & 0 \\
\hline 37 & 8 & B & 7 & 29 & 1 & 1 & 1 & 1 \\
\hline
\end{tabular}

No. number of examinations, FI time interval from first consultation to first imaging, $C S$ time interval to completion of tumor staging, Sono sonography, Comb combination of sonography and computed tomography

${ }^{a}$ Number of imaging methods

${ }^{\mathrm{b}}$ Time from first consultation to first imaging

${ }^{\mathrm{c}}$ Time from first consultation to completion of tumor staging

${ }^{\mathrm{d}}$ Sonography

${ }^{\mathrm{e}}$ Combination of CT and sonography 


\section{Group stratification according to the initial imaging method for staging}

\section{Computed tomography as the initial imaging strategy}

Patients who underwent computed tomography of any body region $(n=14,48.3 \%)$ during radiologic staging, had to undergo 3.2 examinations (median value; median $=3$, SD: 1.2) before staging was completed. Median FI accounted for 9 days (mean $=16$, SD: 16 days) and staging was completed after 28 days (median; mean $=30$ days, SD: 18 days).

In contrast, in 15 patients $(51.7 \%)$, the imaging process was conducted without using computed tomography. Those patients underwent 5.4 investigations (mean value; median $=6$, SD: 2). FI was 31 days (median; mean $=31$, SD: 25 days) and CS was completed after 36 days (mean $=43$ days, SD: 22 days). (Table 3 ).

When patients $(n=8,21.6 \%)$ received sonography of one or more lymph nodes without computed tomography, the mean number of examinations was 4.5 (median $=4$, SD: 2). FI was done after 24 days (median, mean $=26$, SD: 17 days) and CS was completed after 35 (mean $=37$ days, SD: 13 days) (Table 3).

\section{Combination of lymph-node sonography and computed tomography as primary imaging modality}

Patients who received a combination of computed tomography and lymph-node sonography $(n=15)$ underwent a mean of 5.8 examinations per patient (median $=6, \mathrm{SD}: 1.6$ ). The time from first presentation to first imaging was 31 days (median value; mean $=35$, SD: 28 days). $\mathrm{CS}$ was completed within 40 days (median value; mean $=46$ days, SD: 25 days) (Table 3).

\section{Use of PET or PET-CT as staging strategy}

Those patients $(n=7)$ who had a PET or PET-CT underwent a mean number of investigations of 5.3 (median $=5$, SD: 2 days). FI and CS accounted for 11 (mean $=15$, SD: 14 days) and 29 (mean $=31$ days, SD: 14 days), respectively (Table 3).

\section{Sensitivity and specificity of conducted examinations}

After re-evaluation of all imaging methods, tumor staging was changed in $13.5 \%$ of all patients $(n=5)$. In one case, computed tomography showed false-positive, and in two diagnostic imaging of patients suffering from Merkel cell carcinoma according to group and imaging method
Table 3 Time frames for

\begin{tabular}{|c|c|c|}
\hline Modality & $\mathrm{FI}^{\mathrm{a}}$ & $\mathrm{CS}^{\mathrm{a}}$ \\
\hline \multicolumn{3}{|l|}{ Group A } \\
\hline Median & 15 & 35 \\
\hline SD & 16 & 17 \\
\hline Mean & 19 & 31 \\
\hline \multicolumn{3}{|l|}{ Group B } \\
\hline Median & 29 & 43 \\
\hline SD & 27 & 24 \\
\hline Mean & 32 & 45 \\
\hline \multicolumn{3}{|l|}{ CT } \\
\hline Median & 9 & 28 \\
\hline SD & 16 & 18 \\
\hline Mean & 16 & 30 \\
\hline \multicolumn{3}{|c|}{ Sonography } \\
\hline Median & 24 & 35 \\
\hline $\mathrm{SD}$ & 17 & 13 \\
\hline Mean & 26 & 37 \\
\hline \multicolumn{3}{|c|}{ Combination } \\
\hline Median & 31 & 40 \\
\hline SD & 28 & 25 \\
\hline Mean & 35 & 46 \\
\hline \multicolumn{3}{|l|}{ PET-CT } \\
\hline Median & 11 & 29 \\
\hline SD & 2 & 14 \\
\hline Mean & 15 & 31 \\
\hline
\end{tabular}

Group A patients who underwent less than five examinations, Group $B$ patients who underwent five or more examinations, $F I$ time interval from first consultation to first imaging, $C S$ time interval to completion of tumor staging

${ }^{a}$ Values represent time frames in days

cases, false-negative dissemination of the primary tumor, with a sensitivity of $80 \%$ and a specificity of $95 \%$. Similar rates could be determined for sonography with a rate of $82 \%$ for sensitivity and 100\% for specificity (Table 4 ).

\section{Cost-effectiveness of conducted examinations}

Neither use of computed tomography or sonography, nor the combination of those modalities did influence cost-effectiveness significantly $(p=0.48)$. Not surprisingly, use of four examinations or less led to significantly less costs than use of more than four examinations $(p=0.001)$. 
Table 4 Data of false-positive and false-negative results of imaging methods in Merkel cell carcinoma according to imaging modality

\begin{tabular}{|c|c|c|c|c|}
\hline Modality & Disease & Staging & Re-staging ${ }^{\mathrm{a}}$ & $\mathrm{By}^{\mathrm{b}}$ \\
\hline \multirow[t]{3}{*}{$\mathrm{CT}$} & $\begin{array}{l}\text { Pulmonary } \\
\text { metastasis }\end{array}$ & T1N0M1 & T1N0 M0 & PET-CT \\
\hline & $\begin{array}{l}\text { Pelvic lymph } \\
\text { nodes }\end{array}$ & TxN0M0 & TxN1M0 & biopsy \\
\hline & $\begin{array}{l}\text { Cervical lymph } \\
\text { nodes }\end{array}$ & T1N0M0 & T1N1M0 & PET-CT \\
\hline \multirow[t]{2}{*}{ Sonography } & $\begin{array}{l}\text { Inguinal lymph } \\
\text { nodes }\end{array}$ & TxNOM0 & TxN2M0 & CT \\
\hline & $\begin{array}{l}\text { Inguinal lymph } \\
\text { nodes }\end{array}$ & T1N0N0 & T1N1M0 & sonography \\
\hline
\end{tabular}

${ }^{\text {a }}$ Re-staging after re-evaluation by another diagnostic tool

${ }^{\mathrm{b}}$ Imaging method or diagnostic tool that led to correct staging

\section{Discussion}

The key role of imaging in patients suffering from MCC is staging of the tumor by determining the size and localization of the primary lesion and identifying loco-regional invasion including lymph nodes and soft tissue [7, 17, 18]. In the current literature, sonography, computed tomography, and PET $-\mathrm{CT}$ are discussed as the major diagnostic tools in patients with MCC. In this study, we evaluated these three imaging methods to determine their efficacy and accessibility. To our knowledge, this is the first study to show that the choice of the imaging method influences staging success and time until treatment onset. Time delay in staging should be avoided, as it is not only cost-intensive, but also a significant contributor to postponed onset of treatment, being associated with a significantly worse outcome and decreased healthrelated quality of life during waiting times $[2,13]$.

In our study, the staging work-up was conducted with a mean number of 4.5 radiologic examinations. We clearly acknowledge that a lower number of imaging lead to shorter duration of the staging process and, subsequently, to an early initiation of therapy.

Use of computed tomography seems to accelerate the staging process, as fewer examinations were necessary for staging work-up (3 vs. 6). Computed tomography is independent of physicians' skills and all scans can be considered by other clinicians, during rounds and in tumor boards. The majority of incorrect results in our cohort occurred in sonographic inguinal lymph-node staging. However, despite the low sample size, lymph-node staging of the cervical lymph nodes seems to be more accurate than for abdominal lymph nodes. Nevertheless, we hypothesize that the difference in CS between CT and ultrasonography indicates that sonography is very accurate in providing fast information regarding locally invasive and/or metastatic Merkel cell carcinoma disease but lacks in regards to lymph-node mapping and reproducibility. Therefore, further examinations and, in most of our patients, CT imaging were needed to complete tumor staging.

A very limited number of MCC patients in our cohort underwent PET and PET-CT imaging. Recent publications claimed the usefulness of PET-CT in staging of MCC [15, 19]. However, due to the high costs of a PET-CT scan and a limited availability compared to in particular CT and ultrasonography, it is not always useful for routine staging of carcinoma patients. In our study, PET or PET-CT did not show any benefit concerning the number of imaging examinations and, of significant importance, a time delay was observed compared to those patients who received computed tomography and ultrasonography until tumor staging was completed. Interestingly, in all patients, PET or PET-CT was never used as the sole diagnostic tool, which challenges the usefulness of this very expensive examination. However, in a few cases, scintigraphy was useful for the exact diagnosis of secondary lesions.

We analyzed cost-effectiveness of all conducted examinations according to the dominant diagnostic method. No significant difference in cost-effectiveness according to the different imaging modalities was detectable. However, this might be contributed to the low patient number included in this study. Notably, the delay in staging and treatment initiation and a consequently prolonged hospitalization period, which results in a multiplication of incurring costs, has not been taken into account in this analysis.

In general, time delay in staging should be avoided, as it is not only very cost-intensive but also a significant issue for postponed onset of treatment. As previously shown by the group of Tsang, delay of treatment initiation is associated with a significantly worse outcome [2] due to the very aggressive, rapidly growing characteristics of this carcinoma. This is consistent with the other studies showing a negative prognostic influence of waiting times on outcome in head and neck squamous cell carcinoma, breast cancer, and non-small lung cancer [10-12]. Moreover, studies showed that carcinoma patients have a significantly decreased health-related quality of life during waiting times for surgery, and this leads to impairment of vitality and mental health [13]. Thus, we propose prioritization by implementation of an elapsed-time-of-waiting system to improve access to imaging for aggressive diseases [20].

\section{Conclusion}

Our study clearly shows that a huge panel of different imaging methods was and is still used for staging in Merkel cell carcinoma patients. This subsequently leads to a prolonged time interval between imaging and treatment onset. We 
could show that the use of computed tomography as the first imaging method poses an advantage for tumor staging as it accelerates staging work-up and reduces the number of further examinations. Therefore, we strongly recommend that CT should initially be used for tumor staging in patients with Merkel cell carcinoma.

Acknowledgements Open access funding provided by Medical University of Vienna.

Funding No financial disclosures.

\section{Compliance with ethical standards}

Conflict of interest The authors declare that they have no conflict of interest.

Open Access This article is distributed under the terms of the Creative Commons Attribution 4.0 International License (http://creativeco mmons.org/licenses/by/4.0/), which permits unrestricted use, distribution, and reproduction in any medium, provided you give appropriate credit to the original author(s) and the source, provide a link to the Creative Commons license, and indicate if changes were made.

\section{References}

1. Toker C (1972) Trabecular carcinoma of the skin. Arch Dermatol 105:107-110

2. Tsang G, O'Brien P, Robertson R et al (2004) All delays before radiotherapy risk progression of Merkel cell carcinoma. Australas Radiol 48:371-375

3. Ngyen BD, MCCullough AE (2002) Imaging of Merkel cell carcinoma. RG 22:367-376

4. Hitchcock CL, Bland KI, Franzini D, Harris B, Copeland EM III (1988) Neuroendocrine (Merkel cell) carcinoma of the skin: its natural history, diagnosis and treatment. Ann Surg 207:201-207

5. O'Connor WJ, Brodland DG (1996) Merkel cell carcinoma. Dermatol Surg 22:262-267

6. Suárez C, Rodrigo JP, Ferlito A, Devanex KO, Rinaldo A (2004) Merkel cell carcinoma of the head and neck. Oral Oncol 40:773-779

7. Peloschek P, Novotny C, Mueller-Mang C et al (2010) Diagnostic imaging in Merkel cell carcinoma: lessons to learn from 16 cases with correlation of sonography, CT, MRI and PET. Eur J Radiol 73:317-323
8. Becker J, Mauch C, Kortmann RD et al (2008) Short german guidelines: Merkel cell carcinoma. JDDG 6:S15-S16

9. Allen PJ, Bowne WB, Jaques DP, Brennan MF, Busam K, Coit DG (2005) Merkel cell carcinoma: prognosis and treatment of patients from a single institution. JCO 23:2300-2309

10. Van Harten MC, de Ridder M, Hamming-Vrieze O, Smeele LE, Balm AJM, van den Brekel MWM (2014) The association of treatment delay and prognosis in head and neck squamous cell carcinoma (HNSCC) patients in a Dutch comprehensive cancer canter. Oral Oncol 50:282-290

11. Brazda A, Estroff J, Euhus D et al (2010) Delays in time to treatment and survival impact in breast cancer. Ann Surg Oncol 17:291-296

12. Myrdal G, Lambe M, Hillerdal G, Lamberg K, Agustsson T, Stahle E (2004) Effect of delays on prognosis in patients with non-small lung cancer. Thorax 59:45-49

13. Visser MR, van Lanschot JJ, van der Velden J, Kloek JJ, Gouma DJ, Sprangers MA (2006) Quality of life in newly diagnosed cancer patients waiting for surgery is seriously impaired. J Surg Oncol 93:571-577

14. Hawryluk EB, O'Regan KN, Sheehy N et al (2013) Positron emission tomography/computed tomography imaging in Merkel cell carcinoma: a study of 270 scans in 97 patients at the Dana-Farber/Brigham and Women's Cancer Center. J Am Acad Dermatol 68:592-599

15. Concannon R, Larcos GS, Veness M (2010) The impact of (18) F-FDG PET-CT scanning for staging and management of Merkel cell carcinoma: results from Westmead Hospital, Sydney, Australia. J Am Acad Dermatol 62:76-84

16. Liao LJ, Lo WC, Hsu WL, Wang CT, Lai MS (2012) Detection of cervical lymph node metastasis in head and neck cancer patients with clinically N0 neck-a meta-analysis comparing different imaging modalities. BMC Cancer 12:236-242

17. Erovic I, Erovic BM (2013) Merkel cell carcinoma: the past, the present, and the future. J Skin Cancer 2013:929364

18. Grandhaye M, Teixeira PG, Henrot P et al (2015) Focus on Merkel cell carcinoma: diagnosis and staging. Skelet Radiol 44:777-786

19. Treglia G, Kakhki VR, Giovanella K, Sadeghi R (2013) Diagnostic performance of fluorine-18-fluorodeoxyglucose positron emission tomography in patients with Merkel cell carcinoma: a systematic review and meta-analysis. Am J Clin Dermatol 14:437-447

20. Eskander A, Devins GM, Freeman J et al (2013) Waiting for thyroid surgery: a study of psychological morbidity and determinants of health associated with long wait times for thyroid surgery. Laryngoscope 123:541-547 